Journal for ImmunoTherapy of Cancer

\section{Campylobacteriosis following immunosuppression for immune checkpoint inhibitor-related toxicity}

To cite: Lee KA, Shaw $\mathrm{H}$, Bataille $\mathrm{V}$, et al. Campylobacteriosis following immunosuppression for immune checkpoint inhibitorrelated toxicity. Journal for ImmunoTherapy of Cancer 2020;8:e000577. doi:10.1136/ jitc-2020-000577

Accepted 08 August 2020
Check for updates

(C) Author(s) (or their employer(s)) 2020. Re-use permitted under CC BY-NC. No commercial re-use. See rights and permissions. Published by BMJ.

${ }^{1}$ Medical Oncology, Royal Marsden NHS Foundation Trust, London, UK

${ }^{2}$ Department of Twin Research and Genetic Epidemiology,

King's College London, London, UK

${ }^{3}$ Medical Oncology, Mount Vernon Cancer Centre, Northwood, UK

${ }^{4}$ Medical Oncology, University College London, London, UK

${ }^{5}$ Dermatology, Mount Vernon Cancer Centre, Northwood, UK

Correspondence to

Dr Karla A Lee;

karla.lee@nhs.net

\section{ABSTRACT}

Five patients receiving checkpoint inhibitor immunotherapy (CPI) under our care across two cancer centers over a 12-month period have subsequently developed campylobacterosis. All had received immune-suppressive treatment for CPI-related colitis in the weeks or months preceding the detection of Campylobacter infection, with negative stool cultures at presentation of CPI-related colitis. The immune-suppression required to treat CPIrelated toxicity may lead to an increased risk of enteric infection within the gut. While the underlying immune and biologic mechanisms are not well understood, perturbation of the gut microbiota is an increasingly recognized factor capable of influencing CPI-mediated immune reconstitution and response to therapy. Clinicians should be aware that worsening of colitic symptoms in patients with a history of treatment for CPI-related colitis may be due to enteric infection, and not simply a relapse/ deterioration of a previously treated CPI-related colitis. Judicious infectious disease evaluation is necessary for patients receiving CPIs as symptoms can mimic immunerelated adverse events (irAEs). Furthermore, the benefits of immune-suppressive therapy for the treatment of presumptive irAEs must be weighed against the possible increased risk for either enteric infection or opportunistic infection. Prospective studies are required to investigate microbiome perturbations, resulting from immunesuppression, and guide future treatment of this patient cohort.

Five patients receiving checkpoint inhibitor immunotherapy (CPI) under our care across two cancer centers over a 12-month period have subsequently developed campylobacterosis. All five patients had received treatment for CPI-related colitis in the weeks or months preceding the detection of Campylobacter infection, with negative stool cultures at presentation of CPI-related colitis ruling out infection as a cause of their initial colitic symptoms. Of these five patients, one received amoxicillin antibiotics at the point of admission with CPI-related colitis; none of the other four had received antibiotics in the 3 months prior to development of campylobacterosis. One patient developed campylobacterosis 3 months after immune-suppressive treatment for CPI-related colitis; the other four developed campylobacterosis within 3 weeks of immune-suppressive treatment for CPIrelated colitis. Microbiology advice was sought for all five patients, and antibiotic treatment for campylobacterosis was advised for three patients. All five subsequently produced Campylobacter-negative stool and recovered from their infection. CPI-related toxicity and the immune-suppression required to treat it may lead to an increased risk of outgrowth of bacteria such as Campylobacter.

Campylobacter infection is one of the most common causes of infectious diarrhea among immunocompetant hosts. ${ }^{1}$ However, many individuals carry Campylobacter as a commensal gut organism with no adverse effects. Campylobacteriosis is a collective term, used for infectious, emerging foodborne disease caused by Campylobacter species comprizing Gram-negative, curved and microaerophilic pathogens.

Both preclinical and clinical evidence now support the association between response to CPI, gut microbiome taxonomic diversity and enrichment of specific gut bacterial taxa, suggesting that some species or consortia provide intrinsic immunemodulating properties. The landmark study by Gopalakrishnan et al demonstrated how broader stool bacterial diversity and higher representation of Ruminococcaceae communities positively influence patients' survival following $\mathrm{CPI}^{2}$ by promoting a strongly immune-reactive microenvironment and lower systemic release of proinflammatory cytokines. ${ }^{3}$ Subsequently, a number of other commensal bacteria have been recognized to play a similar role including Bifidobacteria spp, a Gram-positive genus highly represented within the intestine that facilitates maturation of dendritic cells and increased accumulation of antigen-specific T-cells within the tumor microenvironment. ${ }^{4}$ Similarly, the anaerobic commensal Akkermansia Muciniphila has been 
found in higher abundance in responders to CPI, who display higher peripheral CD4 and CD8 memory T-cell responses to this bacterium. ${ }^{5}$

Sensitivity to CPI therapy is governed by a complex interplay of tumor and host-related determinants. While the underlying immune-biologic mechanisms are poorly understood, perturbation of the gut microbiota may lead to outgrowth of some bacteria in patients treated with immune-suppression for CPI-related toxicity. Disruption of the delicate balance of commensal bacteria in the gut is seen in the setting of dysbiosis, a state characterized by a less diverse and less stable gut microbiome, with outgrowth of potentially pathogenic bacteria. ${ }^{6}$ Such disturbance can lead to impairment of immune responses with breakdown of mucosal barriers, translocation of bacteria to the mesenteric lymph nodes and blood and alteration of the cytokine environment within the gut mucosa leading to a profound inflammatory state with activation of Th17 and effector T cells. ${ }^{7}$ Of medications, it is not just antibiotics that have harmful effects on commensal gut flora. Imhann et al analyzed the composition of the gut microbiome of 1815 proton pump inhibitor (PPI) users compared with nonusers. Individuals taking PPIs were consistently found to have a less healthy gut microbiome. Differences were in line with known changes that predispose to Clostridioides difficile infections. ${ }^{8}$ Of particular relevance to CPI-related toxicity, corticosteroids may also promote a dysbiosis: among a cohort of 640 non-small cell lung cancer (NSCLC) patients treated with CPI, those who had received more than $10 \mathrm{mg}$ /day of corticosteroids continuously had a decreased progression free survival and overall survival, as confirmed by both univariate and multivariate analyses. ${ }^{9}$ Beyond strong anti-inflammatory and immunesuppressive effects, corticosteroids may cause substantial shifts in the gut microbiota-for instance, dexamethasone causes an increase in the abundance of Clostridiales and Lactobacillaceae in murine models. ${ }^{10}$ Moreover, at the point of CPI-related toxicity, corticosteroids are usually combined with a PPI, which may act as a "double-hit" for the commensal flora.

At present, our knowledge remains limited, but it is clear that we must be mindful of the gut microbiota of patients receiving CPI. While knowledge of CPI-related toxicity has grown substantially over the past decade, ${ }^{11}$ the potential for enteric infection as a result of the immune-suppression used to treat CPI-related toxicity has not previously been well documented. A number of reports detailing more serious opportunistic infections in patients treated for CPI-induced toxicity exist in the literature: two case reports of invasive Aspergillus infection following immune-suppression for CPI-related toxicity, ${ }^{12} 13$ a number of case reports and small case series detailing Pneumocystis jirovecii pneumonia (PJP) among patients treated with immune-suppressants for CPI-related toxicity, ${ }^{14-16}$ two cases of severe Mycobacterium tuberculosis and Aspergillus fumigatus in patients treated for CPI-related toxicity. ${ }^{17}$ The term opportunistic infection refers to infections occurring more frequently and/or severely among immunocompromised individuals; as campylobacteriosis occurs commonly among immunocompentant hosts, it cannot be referred to as an opportunistic infection but nonetheless shares many characteristics of opportunistic infections. A group at Memorial Sloan Kettering Cancer Centre retrospectively reviewed medical records of 740 patients with melanoma who received CPIs, reporting serious infection to have occurred in 54 patients $(7.3 \%) .^{18}$ The major risk factor for development of serious infection among these patients was use of immune-suppressive agents, including corticosteroids and infliximab. The risk of serious infection was $13.5 \%$ in patients who received either corticosteroids or infliximab but only $2 \%$ in those who did not. Infections reported include invasive pulmonary aspergillosis $(\mathrm{n}=2), \mathrm{PJP}(\mathrm{n}=3)$, disseminated or facial Herpes zoster $(\mathrm{n}=3)$ and Clostridioides difficile-related diarrhea $(n=10)$ among others. Another group reviewed 167 patients with NSCLC, 32 (19.2\%) of whom developed infectious diseases. Of the 33 infections in 32 patients, 25 were bacterial, 2 were fungal and 6 were viral. Twentyseven of the 32 patients with infections had received corticosteroids during their treatment course. ${ }^{19}$

Clinicians should be aware that worsening of colitic symptoms in patients with a history of treatment for CPIrelated colitis may be due to enteric infection, and not simply a relapse/deterioration of a previously treated CPI-related colitis. Patients receiving CPIs should always have a stool sample collected and sent for microscopy and culture at presentation, even when relapse/deterioration of a previously treated CPI-related colitis seems the most likely diagnosis. Judicious infectious disease evaluation is necessary for patients receiving CPIs as symptoms can mimic immune-related adverse events (irAEs). Furthermore, the benefits of immune-suppressive therapy for the treatment of presumptive irAEs must be weighed against the possible increased risk for either opportunistic infections or enteric infection. Our current study Predicting Response to Immunotherapy for Melanoma With Gut Microbiome and Metabolomics (PRIMM) (NCT03643289) is assessing changes in gut microbiome differences in patients with melanoma at baseline and while on treatment with CPI in both metastatic and adjuvant settings and will endeavor to determine whether baseline microbiome changes exist among those patients who go on to develop toxicity \pm enteric infection and those who do not. More prospective trials are needed to define the optimal immune-suppressive management of CPIrelated toxicity.

Contributors KAL wrote the manuscript. HS, PN and VB were responsible for the concept. All authors approved the final manuscript.

Funding The authors have not declared a specific grant for this research from any funding agency in the public, commercial or not-for-profit sectors. KAL is supported by the Seerave Foundation and CDRF.

Competing interests PN has consulted for AstraZeneca, BMS, Merck, MSD, Immunocore, Pfizer, Ipsen, 4SC, Pierre Fabre and Roche. HS has consulted for Novartis, MSD, BMS and Sanofi-Genzyme.

Patient consent for publication Not required. 
Provenance and peer review Not commissioned; externally peer reviewed.

Open access This is an open access article distributed in accordance with the Creative Commons Attribution Non Commercial (CC BY-NC 4.0) license, which permits others to distribute, remix, adapt, build upon this work non-commercially, and license their derivative works on different terms, provided the original work is properly cited, appropriate credit is given, any changes made indicated, and the use is non-commercial. See http://creativecommons.org/licenses/by-nc/4.0/.

\section{REFERENCES}

1 Kaakoush NO, Castaño-Rodríguez N, Mitchell HM, et al. Global epidemiology of Campylobacter infection. Clin Microbiol Rev 2015;28:687-720.

2 Gopalakrishnan V, Spencer CN, Nezi L, et al. Gut microbiome modulates response to anti-PD-1 immunotherapy in melanoma patients. Science 2018;359:97-103.

3 Chaput N, Lepage P, Coutzac C, et al. Baseline gut microbiota predicts clinical response and colitis in metastatic melanoma patients treated with ipilimumab. Ann Oncol 2019;30:2012.

4 Sivan A, Corrales L, Hubert N, et al. Commensal Bifidobacterium promotes antitumor immunity and facilitates anti-PD-L1 efficacy. Science 2015;350:1084-9.

5 Routy B, Le Chatelier E, Derosa L, et al. Gut microbiome influences efficacy of PD-1-based immunotherapy against epithelial tumors. Science 2018;359:91-7.

6 Frosali S, Pagliari D, Gambassi G, et al. How the intricate interaction among Toll-like receptors, microbiota, and intestinal immunity can influence gastrointestinal pathology. J Immunol Res 2015;2015:1-12.

7 Levy M, Kolodziejczyk AA, Thaiss CA, et al. Dysbiosis and the immune system. Nat Rev Immunol 2017;17:219-32.

8 Imhann F, Bonder MJ, Vich Vila A, et al. Proton pump inhibitors affect the gut microbiome. Gut 2016;65:740-8.

9 Arbour KC, Mezquita L, Long N, et al. Impact of baseline steroids on efficacy of programmed cell death-1 and programmed Death-Ligand
1 blockade in patients with non-small-cell lung cancer. J Clin Oncol 2018;36:2872-8.

10 Huang EY, Inoue T, Leone VA, et al. Using corticosteroids to reshape the gut microbiome: implications for inflammatory bowel diseases. Inflamm Bowel Dis 2015;21:963-72.

11 Lomax AJ, Lim J, Cheng R, et al. Immune toxicity with checkpoint inhibition for metastatic melanoma: case series and clinical management. J Skin Cancer 2018;2018:1-13.

12 Kyi C, Hellmann MD, Wolchok JD, et al. Opportunistic infections in patients treated with immunotherapy for cancer. $J$ Immunother Cancer 2014;2:19.

13 Lord JD, Hackman RC, Moklebust A, et al. Refractory colitis following anti-CTLA4 antibody therapy: analysis of mucosal Foxp3+ T cells. Dig Dis Sci 2010;55:1396-405.

14 Arriola E, Wheater M, Krishnan R, et al. Immunosuppression for ipilimumab-related toxicity can cause pneumocystis pneumonia but spare antitumor immune control. Oncoimmunology 2015;4:e1040218.

15 Schwarz M, Kocher F, Niedersuess-Beke D, et al. Immunosuppression for immune checkpoint-related toxicity can cause Pneumocystis jirovecii pneumonia (PJP) in non-smallcell lung cancer (NSCLC): a report of 2 cases. Clin Lung Cancer 2019;20:e247-50.

16 Si S, Erickson K, Evageliou N, et al. An usual presentation of pneumocystis jirovecii pneumonia in a woman treated with immune checkpoint inhibitor. J Pediatr Hematol Oncol 2020. doi:10.1097/ MPH.0000000000001757. [Epub ahead of print: 24 Feb 2020].

17 Inthasot V, Bruyneel M, Muylle I, et al. Severe pulmonary infections complicating nivolumab treatment for lung cancer: a report of two cases. Acta Clin Belg 2020;75:1-3.

18 Del Castillo M, Romero FA, Argüello E, et al. The spectrum of serious infections among patients receiving immune checkpoint blockade for the treatment of melanoma. Clin Infect Dis 2016;63:1490-3.

19 Fujita K, Kim YH, Kanai O, et al. Emerging concerns of infectious diseases in lung cancer patients receiving immune checkpoint inhibitor therapy. Respir Med 2019;146:66-70. 